\title{
Effect of Akti-2 on sperm motility, capacitation and acrosome reaction in a mouse model
}

\author{
YANMEI QUAN and QIANG LIU
}

\begin{abstract}
Shanghai Key Laboratory of Reproductive Medicine, Department of Anatomy, Histology and Embryology,
\end{abstract} Faculty of Basic Medicine, School of Medicine, Shanghai Jiao Tong University, Shanghai 200025, P.R. China

Received November 23, 2015; Accepted February 9, 2016

DOI: $10.3892 /$ br.2016.627

\begin{abstract}
The aim of the present study was to investigate the effect of the Akt inhibitor, Akti-2, on the sperm motility and acrosome reaction in mice. Mature sperms from the adult mice, aged 8 weeks, were co-incubated with Akti-2 for $\sim 30 \mathrm{~min}$ at $37^{\circ} \mathrm{C}$ in $5 \% \mathrm{CO}_{2}$, and the sperm viability was assessed by eosin-nigrosin staining. The sperm total and progressive motility were analyzed by computer-aided sperm analysis. In addition, the acrosome reaction of sperms was detected by the acid phosphatase assay, Coomassie Brilliant Blue staining and fluorescein-isothiocyanate conjugated pisum sativum lectin staining, respectively. Compared with the control (dimethyl sulfoxide), Akti-2 had no effect on sperm viability, but it suppressed the total and progressive motility significantly. Furthermore, the capacitation-associated protein tyrosine phosphorylation and the acrosome reaction induced by calcium ionophore A23187 could be suppressed by Akti-2. These experiments confirmed that Akti-2 significantly impaired the sperm functions, including motility, capacitation and acrosome reaction, and provide the proof for its potential in male reproductive toxicity.
\end{abstract}

\section{Introduction}

The phosphoinositide 3-kinase (PI3K)/Akt signaling pathway has an extremely important function on cell proliferation, cell metabolism and angiogenesis. Abnormalities of the pathway were tested in numerous tumor cell lines. The $\mathrm{PI} 3 \mathrm{~K} / \mathrm{Akt}$ pathway is closely associated with the pathogenesis, progression and metastasis of carcinoma (1). Two types of

Correspondence to: Professor Qiang Liu, Shanghai Key Laboratory of Reproductive Medicine, Department of Anatomy, Histology and Embryology, Faculty of Basic Medicine, School of Medicine, Shanghai Jiao Tong University, 280 South Chongqing Road, Shanghai 200025, P.R. China

E-mail: qliu0122@shsmu.edu.cn

Key words: Akti-2, acrosome reaction, sperm motility, sperm viability, sperm capacitation
PI3K inhibitors have been widely studied, Wortmannin and LY294002. However, these inhibitors are confined to in vitro research due to their potent toxicity in vivo.

Akt is a serine/threonine kinase that belongs to the AGC family of kinases (2) and shares high homology with protein kinase A (PKA) and PKC. Akt is also known as PKB, and there are three mammalian isoforms of this enzyme: Akt-1, Akt-2 and Akt-3. Furthermore, the three isozymes of Akt share $>85 \%$ homology and all possess an amino terminal pleckstrin homology domain and a kinase domain separated by a 39 -amino acid hinge region $(3,4)$.

Akt is a critical downstream effector of multiple growth factors and receptors involved in tumorgenesis, and could also regulate a number of downstream targets $(5,6)$. When Akt was inhibited, a number of its targeted proteins are suppressed, and this may delay tumor progression. As a result, studies on Akt inhibitors have focused on the field of tumor therapy $(7,8)$. Akt-1 and Akt-2 are commonly overexpressed or constitutively active in a large number of human cancers including brain, gastric, colon, breast, lung and prostate carcinomas, and their activation correlates to cancer progression (4). Akti-2, a selective allosteric inhibitor of Akt-1 and -2, was previously shown to be effective in prostate cancer and other solid tumors (8). MK-2206, an allosteric Akt inhibitor, has progressed into a phase II clinical trial. The present study examined the effect of Akti-2 treatment on sperm viability, sperm motility, capacitation and acrosome reaction induced by calcium ionophore A23187 in mice, and it may assist the elucidation of the clinical candidate of MK-2206 (Merck \& Co., Inc., Kenilworth, NJ, USA).

\section{Materials and methods}

Materials and reagents. A total of 40 male C57BL/6 mice (8 weeks old) were purchased from the Shanghai Laboratory Animal Center (Shanghai, China), and acclimated in the Animal Center of Shanghai Jiao Tong University School of Medicine (Shanghai, China) for 1 week prior to initiating the study. The study protocol was approved by the Shanghai Jiao Tong University School of Medicine Institutional Animal Care and Use Committee.

The Akt inhibitor Akti-2 was from Merck Millipore (Billerica, MA, USA), VectaMount permanent mounting medium was from Vector Laboratories, Inc. (Burlingham, 
CA, USA), and Commassie Blue G-250 was from Amresco (Solon, OH, USA). All other chemicals were purchased from Sigma-Aldrich (St. Louis, MO, USA).

Preparation of mouse sperm and capacitation. Mouse sperm was obtained by cutting the isolated cauda epididymis of mature C57BL/6 mice under HM buffer, which is a modified Krebs-Ringer bicarbonate medium (HM-HEPES buffered) (9). The sperm suspension was transferred to a new tube after a 10 -min incubation at $37^{\circ} \mathrm{C}$ in $5 \% \mathrm{CO}_{2}$ and analyzed by computer-aided sperm analysis (CASA; CEROS; Hamilton Thorne, Beverly, MA, USA). The samples were washed 3 times with HM by centrifugation at $500 \mathrm{x}$ g for $5 \mathrm{~min}$. To capacitate mouse sperm, the sperm pellet was resuspended in the modified Krebs-Ringer bicarbonate medium (HMB, which is HM buffer with bicarbonate and bovine serum albumin) (9), adjusted to $1 \times 10^{6}$ sperm $/ \mathrm{ml}$, and incubated for $1 \mathrm{~h}$ at $37^{\circ} \mathrm{C}$ in $5 \% \mathrm{CO}_{2}$. Sperm motility was checked several times in each experiment in a $20-\mu 1$ sperm suspension viewed by CASA to identify the number of living cells. Experiments in which the motility of the fraction of living cells was $<70 \%$ were not considered.

Detection of the sperm capacitation-associated tyrosine phosphorylation. Spermatozoa incubated at capacitation conditions for various periods of time with either dimethyl sulfoxide (DMSO) or $50 \mu \mathrm{M}$ Akti-2 were collected by centrifugation at $5,000 \mathrm{x}$ g for $2 \mathrm{~min}$ (10). Subsequent to washing with $1 \mathrm{ml}$ of phosphate-buffered saline (PBS), the spermatozoa pellet was resuspended in SDS-PAGE sample buffer and boiled for $5 \mathrm{~min}$. Western blot analysis was performed with $10 \%$ SDS-PAGE and proteins were transferred to a Hybond P $0.45 \mu \mathrm{M}$ PVDF membrane (Amersham, GE Healthcare Life Sciences, Logan, UT, USA). The membrane was blocked by incubating with $5 \%$ bovine serum albumin for $1 \mathrm{~h}$ at room temperature. Subsequently, the membrane was immunoblotted with a monoclonal antibody against phosphotyrosine (clone 4G10; Merck Millipore) for $1 \mathrm{~h}$ at room temperature and with horseradish peroxidase (HRP)-conjugated secondary antibodies for $1 \mathrm{~h}$ at room temperature. Detection of the bound antibody was performed using Immobilon Western HRP Substrate (Merck Millipore). $\alpha$-tubulin was used as the internal control.

Induction of the acrosome reaction. Following capacitation, $10 \mu \mathrm{M}$ calcium ionophore $\mathrm{A} 23187$ was added to induce the acrosome reaction in the suspension. In different sets of experiments, the following reagents were added to the sperm suspension following capacitation: $10 \mu \mathrm{M}$ A23187 or $10 \mu \mathrm{M}$ $\mathrm{A} 23187+50 \mu \mathrm{M}$ Akti-2. The control groups were assayed with the addition of the vehicle, DMSO.

Acid phosphatase activity assay. The suspension of sperm following the acrosome reaction was centrifuged at $500 \mathrm{x} \mathrm{g}$ for $5 \mathrm{~min}$, and the supernatant was placed on ice. The activity of the enzymes was measured fluorometrically (11) using the corresponding 4-methylumbelliferyl as a substrate at a concentration of $0.03 \mathrm{mg} / \mathrm{ml}$ in $0.05 \mathrm{~mol} / 1$ citrate buffer ( $\mathrm{pH} 4.5$ ). After incubation for $1 \mathrm{~h}$ at $37^{\circ} \mathrm{C}$, the reaction was stopped by adding the same volume of $0.4 \mathrm{~mol} / \mathrm{l}$ glycine buffer (pH 10.4). The enzyme activity was assayed by luminescence spectrometer (LS50B; Perkin Elmer, Inc., Waltham, MA,
USA) at excitation/emission: $365 / 449 \mathrm{~nm}$. One unit of activity represents the amount of enzyme that catalyzes the release of $1 \mathrm{nmol}$ of 4 -methylumbelliferone $/ \mathrm{ml} / \mathrm{h}$ (12). The culture medium, HM, was used as the blank to zero the spectrofluorometer. Medium plus ionosphere, HMB media and HMB plus Akti-2 were checked for any fluorescence signal, but no difference was observed (data not shown).

Coomassie Brilliant Blue staining. The suspension of the reacted sperm acrosome was centrifuged at $800 \mathrm{x}$ g for $5 \mathrm{~min}$, and the supernatant was removed. The sperm pellet was resuspended in $10 \mu \mathrm{l}$ PBS, smeared and dried in the air. The sperm was fixed in $4 \%$ paraformaldehyde for $10 \mathrm{~min}$, and subsequently washed by PBS three times for $5 \mathrm{~min}$, and $0.1 \mathrm{~mol} / \mathrm{l}$ ammonium acetate $(\mathrm{pH} 9.0)$ twice for $5 \mathrm{~min}$. Following this, the slides were dried in the air, stained by $0.22 \%$ Coomassie Brilliant Blue for $2 \mathrm{~min}$ and washed with flowing water. The slides were mounted with cover slips under a microscope (ECLIPSE E600; Nikon, Tokyo, Japan) and $\geq 200$ sperm were scored as 'acrosome intact' or 'acrosome reacted' (13).

Fluorescein-isothiocyanate conjugated pisum sativum lectin (PSA-FITC) staining. The sperm that had reacted were smeared, dried onto microscope slides and fixed with $4 \%$ paraformaldehyde at room temperature for $10 \mathrm{~min}$. The percentage of acrosome-reacted cells was evaluated using PSA-FITC, as previously described (14). Briefly, smeared spermatozoa were incubated with PSA-FITC $(100 \mu \mathrm{g} / \mathrm{ml})$ in the dark for $30 \mathrm{~min}$. The slides were washed with PBS, and subsequently incubated with $100 \mu \mathrm{g} / \mathrm{ml}$ propidium iodide (Sigma-Aldrich) in the dark for $5 \mathrm{~min}$. The slides were viewed under a laser scanning confocal microscope (LSM-510; Carl Zeiss, Jena, Germany).

Eosin-nigrosin test. Sperm viability was measured using the eosin-nigrosin test. Briefly, a $10-\mu 1$ sperm suspension in $\mathrm{HM}$ and $10 \mu \mathrm{l}$ eosin-nigrosin solution were mixed for $30 \mathrm{sec}$ at room temperature, and smeared onto a slide and allowed to air-dry (15). Slides were subsequently mounted with a coverslip using VectaMount permanent mounting medium (Vector Laboratories, Inc.). Measurements were performed on 200 sperm counted on each slide under the microscope. The percentage of unstained (white) and stained (pink) sperm was calculated. Only the unstained sperm were considered viable.

CASA. A $20-\mu 1$ sperm suspension was tested by CASA and at least five fields of $\sim 1,000$ sperms randomly were detected. This indicated the sperm concentration, motility of total motile and progressive motile sperm. The total motile sperm, which reached $70 \%$, was qualified.

Statistical analysis. Quantitative data are expressed as mean \pm standard deviation. All the analyses were performed using SPSS 18.0 software and GraphPad Software (Graph Pad Software, Inc., La Jolla, CA, USA). P $<0.05$ was considered to indicate a statistically significant difference.

\section{Results}

Sperm viability is not affected by Akti-2. In the different sets of experiments, the following reagents were added into the sperm 


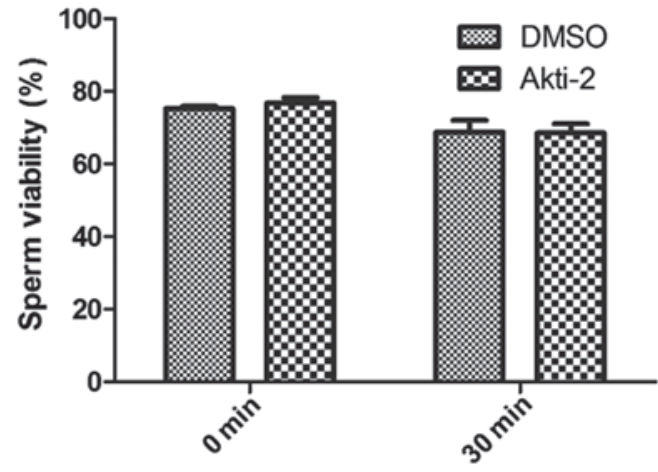

Figure 1. Effect of Akti-2 on sperm viability. Sperm viability after co-incubation with $50 \mu \mathrm{M}$ Akti-2 in $\mathrm{HM}$ buffer at $37^{\circ} \mathrm{C}$ in $5 \% \mathrm{CO}_{2}$ for $30 \mathrm{~min}$ was tested, and the DMSO treatment only was the control. Each bar represents the mean \pm standard deviation of $\geq 4$ experiments. DMSO, dimethyl sulfoxide.

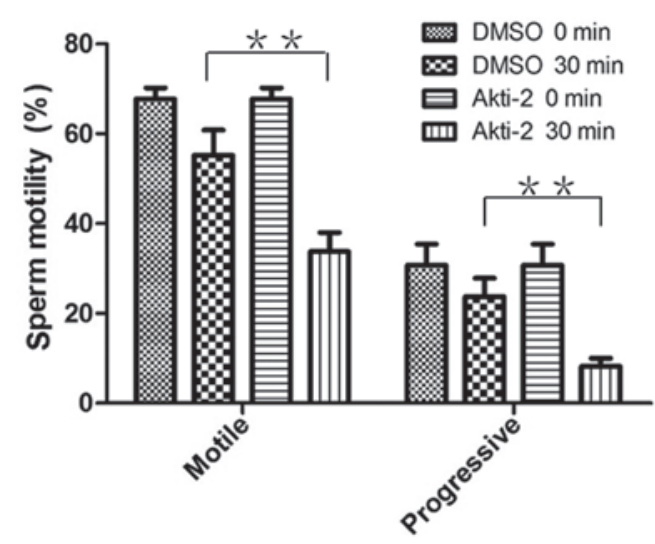

Figure 2. Effects of Akti-2 on sperm viability. Total motile and progressive motile sperm were analyzed with $50 \mu \mathrm{M}$ Akti-2 or DMSO (control) in HM buffer at $37^{\circ} \mathrm{C}$ in $5 \% \mathrm{CO}_{2}$ for $30 \mathrm{~min}$. Compared with the control (total motile sperm, 55.3\%; progressive motile sperm, 23.8\%), Akti-2 suppressed the sperm motility significantly (total motile sperm, $33.7 \%$; progressive motile sperm, $8.2 \%)\left({ }^{* *} \mathrm{P}<0.01\right)$. DMSO, dimethyl sulfoxide.

suspension: Akti-2 $(50 \mu \mathrm{M})$ and the same volume of DMSO as the control, with incubation at $37^{\circ} \mathrm{C}$ and $5 \% \mathrm{CO}_{2}$ for $30 \mathrm{~min}$. The sperm were stained by eosin-nigrosin solution and the sperm viability was detected. The sperm viability of Akti-2-treated sperm at 0 min was $76.8 \pm 3.1 \%$, while after Akti- 2 treatment for $30 \mathrm{~min}$, the sperm viability was $68.5 \pm 5.0 \%$. Compared with the control group (75.3 $\pm 1.2 \%$ at $0 \mathrm{~min}$ and $68.8 \pm 6.6 \%$ at $30 \mathrm{~min})$, there was no difference in the sperm viability between the groups with added Akt inhibitor Akti-2 and the control (P>0.05; Fig. 1).

Akti-2 inhibits sperm motility. After co-incubating with $50 \mu \mathrm{M}$ Akti-2 in $\mathrm{HM}$ at $37^{\circ} \mathrm{C}$ and $5 \% \mathrm{CO}_{2}$ for $30 \mathrm{~min}$, sperm motility was detected. After co-incubation for $30 \mathrm{~min}$, the total motile sperm in the control (DMSO treatment only) was $55.3 \pm 11.1 \%$, and the progressive motile sperm was $23.8 \pm 8.1 \%$. The total motile sperm for the Akti-2 treatment was only $33.7 \pm 8.5 \%$, and the progressive motile sperm was $8.2 \pm 3.6 \%$. Compared with the control, Akti-2 inhibited the sperm motility significantly ( $\mathrm{P}<0.01$; Fig. 2).

Akti-2 inhibits the sperm capacitation. The protein tyrosine phosphorylation event has been associated with sperm

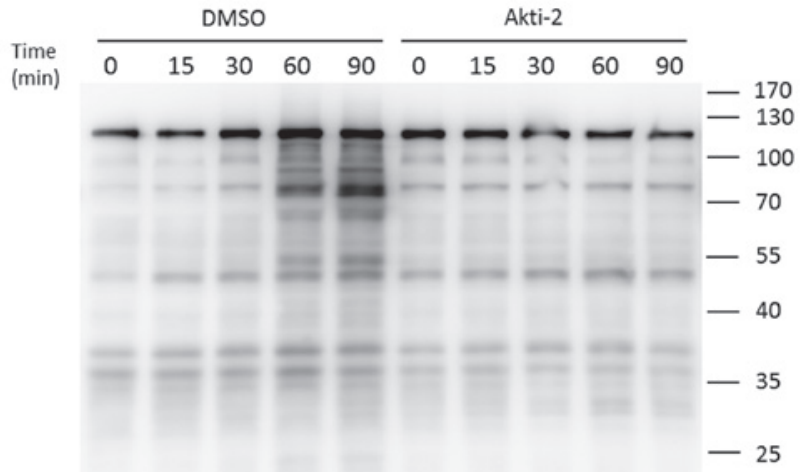

$\alpha$-tubulin

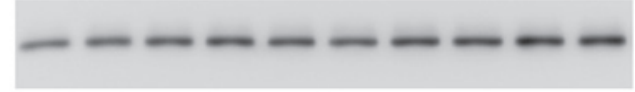

Figure 3. Akti-2 inhibits the sperm capacitation-associated tyrosine phosphorylation. Sperm $\left(2 \times 10^{6} / \mathrm{ml}\right)$ were incubated in HMB for different times with or without Akti-2 at $37^{\circ} \mathrm{C}$ in $5 \% \mathrm{CO}_{2}$, respectively. Protein tyrosine phosphorylation was assessed by western blot analysis. $\alpha$-tubulin was used as the loading control. DMSO, dimethyl sulfoxide.

capacitation and is considered a hallmark event of capacitation (10). Fig. 3 shows a time-dependent increase in sperm capacitation-associated tyrosine phosphorylation when the spermatozoa were incubated with DMSO during capacitation. By contrast, Akti-2 significantly inhibited the time-dependent tyrosine phosphorylation during capacitation.

Akti-2 inhibits the sperm acrosome reaction. The sperm acrosome reaction was detected using the acid phosphatase activity assay and PSA-FITC staining, and the results were confirmed by the traditional Coomassie Brilliant Blue staining (Fig. 4).

Compared with the group with only calcium ionophore A23187 treatment, the acrosome reaction of the group with additional Akti-2 treatment decreased to the level of the DMSO treatment group. In the acid phosphatase activity assay, the acrosome reaction in the Akti-2 treated sperm decreased $18 \%$ compared to the acrosome reaction induced by A23187 treatment only. By Coomassie Brilliant Blue staining, the acrosome reaction in the Akti-2 treatment sperm decreased to almost half of the A23187 only treatment (40.9 and 73.9\%, respectively). Therefore, Akt inhibitor Akti-2 suppressed the sperm acrosome reaction induced by A23187 ( $<<0.05$; Fig. 3).

Akti-2 inhibits the sperm acrosome reaction time-dependently. The acrosome reaction was detected at the time when A23187 and Akti- 2 were added $(0 \mathrm{~min})$ and at 10,20 and $30 \mathrm{~min}$ respectively. The results showed that Akti-2 significantly inhibited the acrosome reaction induced by A23187 as soon as co-incubation for $10 \mathrm{~min}$. Therefore, Akti-2 inhibition is time-dependent ( $\mathrm{P}<0.05$; Fig. 5).

\section{Discussion}

The tumor incidence has been increasing significantly and tumors have become more common and a frequently occurring disease in recent years due to factors such as environmental 
A

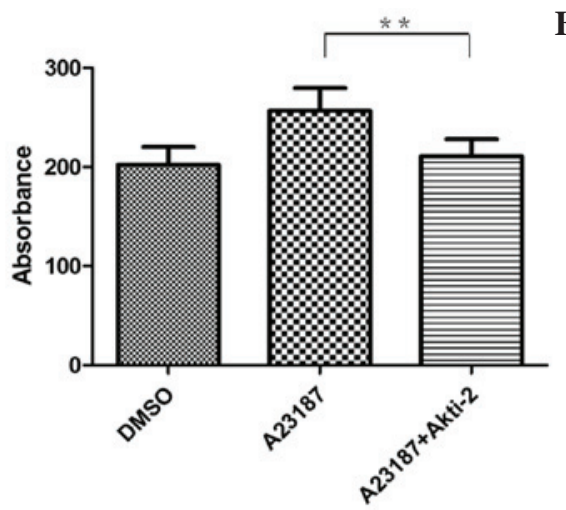

B

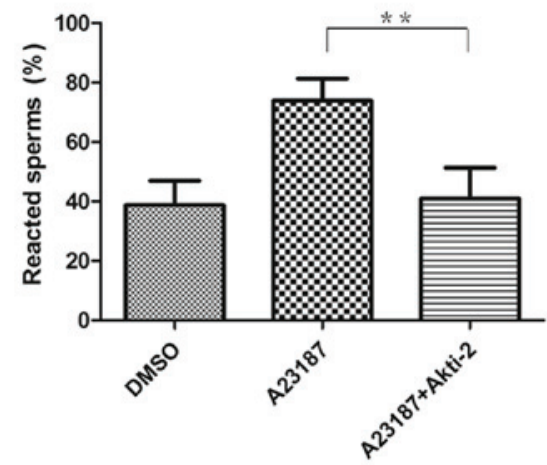

C
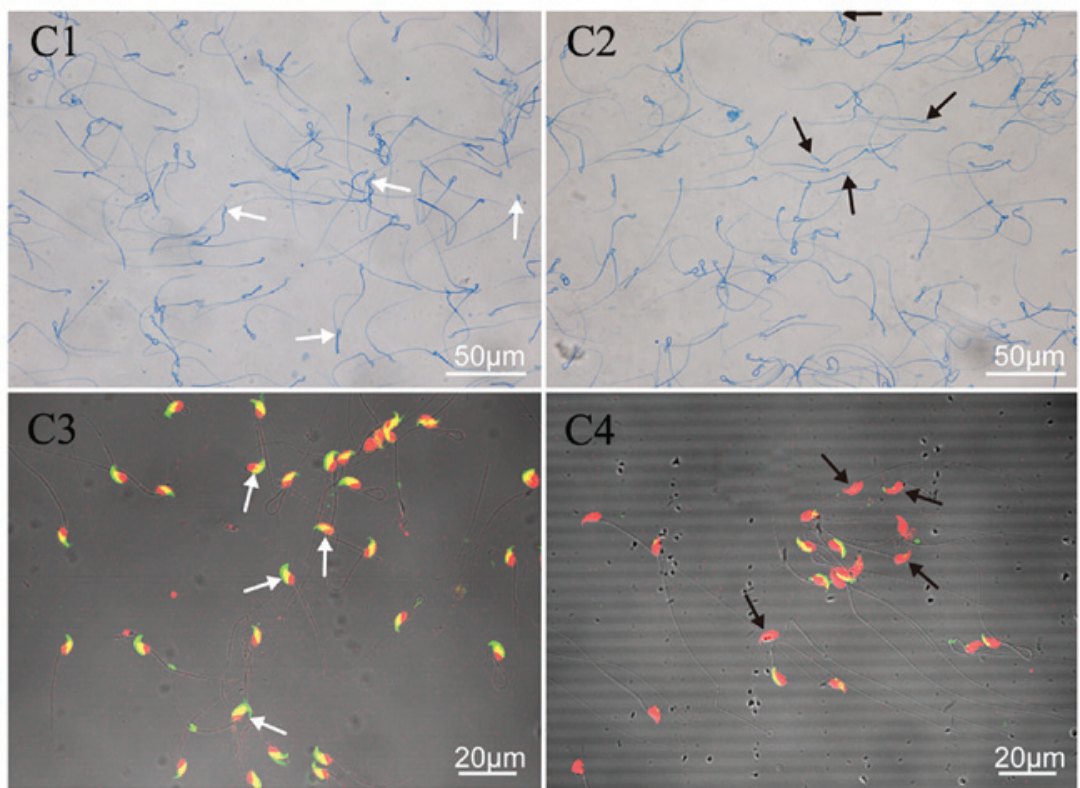

Figure 4. Akti-2 inhibits the sperm acrosome reaction. Detection of inhibition of Akti-2 on the sperm acrosome reaction. Akti-2 inhibited the sperm acrosome reaction induced by $\mathrm{A} 23187$ when co-incubated in $\mathrm{HMB}$ buffer for 30 min at $37 \geq \mathrm{C}$ in $5 \% \mathrm{CO}_{2}$, by the (A) acid phosphatase activity assay $\left.{ }^{(* *} \mathrm{P}<0.01\right)$ and (B) Coomassie Brilliant Blue staining $\left.{ }^{(* *} \mathrm{P}<0.01\right)$. (C) The acrosome reaction induced by A23187 was reduced with Akti-2 (C1 and C3), compared with A23187 only (C2 and $\mathrm{C} 4)$. The white arrows indicate the intact acrosome, and the black arrows indicate acrosome reacted.

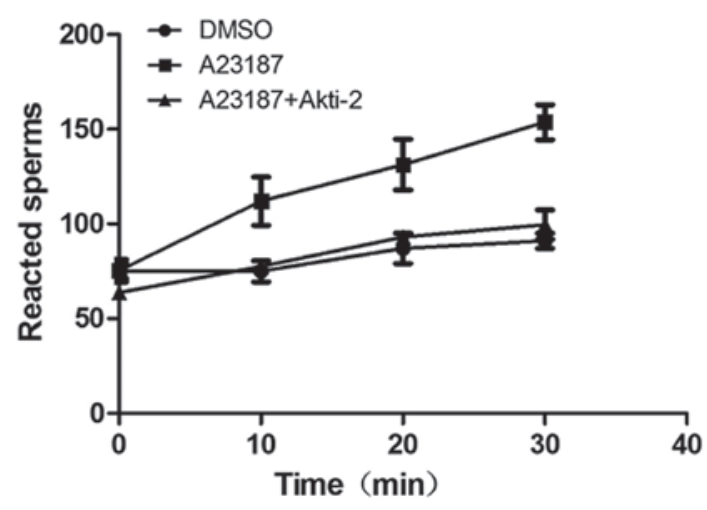

Figure 5. Akti-2 inhibits the sperm acrosome reaction time-dependently. The sperm were co-incubated with Akti-2 in the $\mathrm{HMB}$ buffer at $37^{\circ} \mathrm{C}$ in $5 \% \mathrm{CO}_{2}$ and detected by Coomassie Brilliant Blue staining at 10, 20 or $30 \mathrm{~min}$, respectively. Compared with the control (A23187 only), Akti-2 inhibited the sperm acrosome reaction significantly from $10 \mathrm{~min}$.

pollution, pressure of work and irregular rest. The incidence of tumors is occurring in an increasing number of young people. Akt is a serine/threonine kinase that has recently acquired significant attention as a promising molecular target for cancer therapy due to its critical role (7). It is a homolog of the $\mathrm{t}-8$ strain from the AKR/J mouse (Akt-8), which is known as an oncogene. Akt is overexpressed or overactivated in cancers, including prostate cancer, hepatic carcinoma, gastric carcinoma and oral carcinoma (4). In addition to tumors, Akt is also activated in chronic kidney disease (16). Inhibition of Akt could suppress the tumor progression (17-19). The present study investigated the function of the Akt inhibitor Akti-2 on mouse sperm, and provided information with regards to toxicity of the Akt inhibitor in the therapy for tumor sufferers.

After co-incubation with Akti-2 for 30 min, sperm viability was detected and there was no effect in comparison to the control. However, with regards to sperm motility, Akti-2 inhibited sperm total motility and progressive motility significantly. Furthermore, Akti-2 significantly inhibited the sperm capacitation. Finally, Akti-2 significantly decreased the acrosome reaction induced by $\mathrm{A} 23187$, and the acrosome reaction was reduced after $10 \mathrm{~min}$. As mentioned, Akti-2 has a significant inhibition effect on the reproduction of mice.

With regards to the concentration of Akti-2,50 $\mu \mathrm{M}$ is under the concentration used in the clinical trial (20). However, the 
minimum concentration that could influence the sperm acrosome reaction remains to be elucidated.

Capacitation is necessary for sperm prior to the acrosome reaction (21). An increase in protein tyrosine phosphorylation has been shown to accompany capacitation (22). Another feature in the capacitation is the elevation of sperm $\mathrm{Ca}^{2+}$, which appears to be responsible for an asymmetric form of motility known as hyperactivation. $\mathrm{Ca}^{2+}$ inflow has been investigated previously, and is achieved by Catsper channels in sperm flagellum (23). The effect of Akti-2, an inhibitor of Akt-1/2, which are members of PKB, on the process of capacitation by the $\mathrm{Ca}^{2+}$ signal through the Catsper channels remains to be elucidated, as well as whether Akti-2 affects spermatogenesis or only suppresses the mature sperm. The Akt downstream target protein responsible for sperm motility, capacitation and acrosome reaction and whether it is one target or more remains to be elucidated. Therefore, further studies are required. Analysis using human sperm is also required to confirm these results. This study would aid the application of the Akt inhibitor, clinical candidate MK-2206.

In conclusion, the Akt inhibitor, Akti-2, appeared to suppress the sperm motility, capacitation and acrosome reaction, but not the sperm viability in mice. Thus, Akti-2 may influence the fertility of male patients. Further studies are required to elucidate the exact impact of the Akt inhibitor and to aid in judging the correct application of the clinical candidate, MK2206.

\section{Acknowledgements}

The authors would like to thank Dr Tianyuan $\mathrm{Xu}$ and Dr Xianjin Wang for reading the manuscript and for their technical assistance. The National Natural Science Foundation of China supported the present study (grant nos. 81571488 and 31271600 ).

\section{References}

1. West KA, Castillo SS and Dennis PA: Activation of the PI3K/Akt pathway and chemotherapeutic resistance. Drug Resist Updat 5: 234-248, 2002.

2. Staal SP: Molecular cloning of the akt oncogene and its human homologues AKT1 and AKT2: Amplification of AKT1 in a primary human gastric adenocarcinoma. Proc Natl Acad Sci USA 84: 5034-5037, 1987.

3. Hanks SK and Hunter T: Protein kinases 6. The eukaryotic protein kinase superfamily: Kinase (catalytic) domain structure and classification. FASEB J 9: 576-596, 1995.

4. Zinda MJ, Johnson MA, Paul JD, Horn C, Konicek BW, Lu ZH, Sandusky G, Thomas JE, Neubauer BL, Lai MT and Graff JR: AKT-1, -2 , and -3 are expressed in both normal and tumor tissues of the lung, breast, prostate, and colon. Clin Cancer Res 7: 2475-2479, 2001

5. Jeong SJ, Pise-Masison CA, Radonovich MF, Park HU and Brady JN: Activated AKT regulates NF-kappaB activation, p53 inhibition and cell survival in HTLV-1-transformed cells. Oncogene 24: 6719-6728, 2005.

6. Song G, Ouyang G and Bao S: The activation of Akt/PKB signaling pathway and cell survival. J Cell Mol Med 9: 59-71, 2005.
7. Lindsley CW, Zhao Z, Leister WH, Robinson RG, Barnett SF, Defeo-Jones D, Jones RE, Hartman GD, Huff JR, Huber HE and Duggan ME: Allosteric Akt (PKB) inhibitors: Discovery and SAR of isozyme selective inhibitors. Bioorg Med Chem Lett 15: 761-764, 2005.

8. Cherrin C, Haskell K, Howell B, Jones R, Leander K, Robinson R, Watkins A, Bilodeau M, Hoffman J, Sanderson P, et al: An allosteric Akt inhibitor effectively blocks Akt signaling and tumor growth with only transient effects on glucose and insulin levels in vivo. Cancer Biol Ther 9: 493-503, 2010.

9. Pietrobon EO, Domínguez LA, Vincenti AE, Burgos MH and Fornés MW: Detection of the mouse acrosome reaction by acid phosphatase. Comparison with chlortetracycline and electron microscopy. J Androl 22: 96-103, 2001.

10. Visconti PE, Bailey JL, Moore GD, Pan D, Olds-Clarke P and Kopf GS: Capacitation of mouse spermatozoa. I. Correlation between the capacitation state and protein tyrosine phosphorylation. Development 121: 1129-1137, 1995.

11. Barret AJ and Heath MF: Lysosomal enzymes. In: Lysosomes: A Laboratory Handbook. Dingle JT (ed). 2nd edition. Elsevier/North-Holland Biomedical Press, Amsterdam, pp19-145, 1977.

12. Belmonte SA, Challa A, Gutierrez LS, Bertini F and Sosa MA: alpha-Mannosidase from rat epididymal fluid is a ligand for phosphomannosyl receptors on the sperm surface. Int J Androl 21: 277-282, 1998.

13. Aarons D, Boettger-Tong H, Holt G and Poirier GR: Acrosome reaction induced by immunoaggregation of a proteinase inhibitor bound to the murine sperm head. Mol Reprod Dev 30: 258-264, 1991.

14. Fichorova $\mathrm{R}$ and Anderson DJ: Use of sperm viability and acrosomal status assays in combination with immunofluorescence technique to ascertain surface expression of sperm antigens. J Reprod Immunol 20: 1-13, 1991.

15. Banihani S, Agarwal A, Sharma R and Bayachou M: Cryoprotective effect of L-carnitine on motility, vitality and DNA oxidation of human spermatozoa. Andrologia 46: 637-641, 2014.

16. Lan A and Du J: Potential role of Akt signaling in chronic kidney disease. Nephrol Dial Transplant 30: 385-394, 2015.

17. Sajithlal GB, Hamed HA, Cruickshanks N, Booth L, Tavallai S, Syed J, Grant S, Poklepovic A and Dent P: Sorafenib/regorafenib and phosphatidyl inositol 3 kinase/thymoma viral proto-oncogene inhibition interact to kill tumor cells. Mol Pharmacol 84: 562-571, 2013.

18. Hu C, Dadon T, Chenna V, Yabuuchi S, Bannerji R, Booher R, Strack P, Azad N, Nelkin BD and Maitra A: Combined Inhibition of Cyclin-Dependent Kinases (Dinaciclib) and AKT (MK-2206) Blocks Pancreatic Tumor Growth and Metastases in Patient-Derived Xenograft Models. Mol Cancer Ther 14: 1532-1539, 2015.

19. Conti A1, Santoni M, Amantini C, Burattini L, Berardi R, Santoni G, Cascinu S and Muzzonigro G: Progress of molecular targeted therapies for advanced renal cell carcinoma. Biomed Res Int 2013: 419176, 2013.

20. Gonzalez-Angulo AM, Krop I, Akcakanat A, Chen H, Liu S, Li Y, Culotta KS, Tarco E, Piha-Paul S, Moulder-Thompson S, et al: SU2C phase Ib study of paclitaxel and MK-2206 in advanced solid tumors and metastatic breast cancer. J Natl Cancer Inst 107: pii: dju493, 2015.

21. Fraser LR: Requirements for successful mammalian sperm capacitation and fertilization. Arch Pathol Lab Med 116: 345-350, 1992.

22. Naz RK and Rajesh PB: Role of tyrosine phosphorylation in sperm capacitation/acrosome reaction. Reprod Biol Endocrinol 2: 75, 2004.

23. Suarez SS: Control of hyperactivation in sperm. Hum Reprod Update 14: 647-657, 2008. 